Research Square
Preprints are preliminary reports that have not undergone peer review.

They should not be considered conclusive, used to inform clinical practice, or referenced by the media as validated information.

\title{
Camrelizumab combined with sorafenib versus sorafenib alone in patients with advanced hepatocellular carcinoma: a retrospective study
}

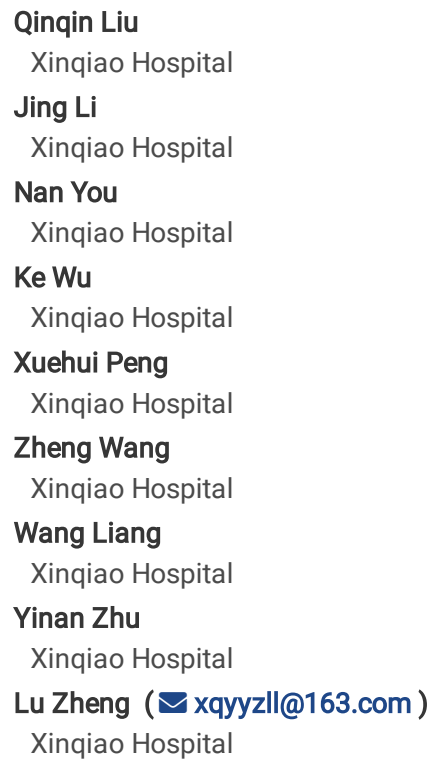




\section{Abstract}

Background: Few studies have evaluated the efficacy and safety of immunotherapy and targeted therapy in combination. The present study aimed to compare camrelizumab plus sorafenib versus sorafenib alone in patients with advanced hepatocellular carcinoma using a propensity score analysis.

Patients and methods: Between January 2019 and January 2021, a total of 100 patients with advanced HCC in the Second Affiliated Hospital of Army Medical University were retrospectively analyzed. Of the patients involved, 35 patients received combined camrelizumab plus sorafenib treatment, and 65 patients received sorafenib monotherapy. After 1:1 propensity score matching (PSM), 34 patients were included in each group. The progression-free survival (PFS), overall survival (OS), treatment response and the relevant adverse effects (AEs) were evaluated.

Results: The combined-therapy group showed significantly improved overall response rate (ORR) than the sorafenib-only group (before PSM, $\mathrm{P}=0.037$; after $\mathrm{PSM}, \mathrm{P}=0.010$ ), but no difference was noted in disease control rate ( $\mathrm{DCR}$ ) (before PSM, $\mathrm{P}=0.695$; after $\mathrm{PSM}, \mathrm{P}=1.000$ ). The median $\mathrm{PFS}$ was significantly longer in the combined-therapy group than the sorafenib-only group (before PSM, $P=0.041$; after $P S M, P=0.043$ ). However, the two groups exhibited comparable median OS (before PSM, $\mathrm{P}=0.135$; after $\mathrm{PSM}, \mathrm{P}=0.105$ ). Although The incidence of thrombocytopenia after PSM was significantly higher in the combined-therapy group than in the sorafenib-only group, most of the AEs could be easily controlled after treatment.

Conclusion: The combination treatment of camrelizumab with sorafenib showed promising efficacy with acceptable safety for the management of advanced HCC.

\section{Introduction}

Hepatocellular carcinoma (HCC), one of the most lethal malignant tumors globally, is the second-ranked cause of cancer death worldwide [1]. HCC is often detected at a late stage due to the insidious and asymptomatic progression, which is not amenable to the curative interventions including liver resection, liver transplantation and radiofrequency ablation [2,3]. Despite tremendous progress in HCC diagnosis and treatment, the clinical outcomes of advanced HCC remain disappointing with the current available therapeutic modalities[4]. Therefore, it is of paramount importance to investigate more effective treatment strategies against advanced HCC.

Multitarget tyrosine kinase inhibitors (TKIs) play a vital role in the clinical management of patients with various kinds of solid tumors [5]. TKIs suppress tumor proliferation and angiogenesis by targeting the vascular endothelial growth factor receptors (VEGFRs), platelet-derived growth factor receptors (PDGFRs), fibroblast growth factor receptors (FGFRs), stem cell factor receptor (KIT) and glial cell-derived neurotrophic factor receptor (RET) [6-8]. Of these, sorafenib is the first-line targeted agent for advanced HCC patients, which achieved a median overall survival (OS) of 6.5-10.7 months and median time to progression of 2.8-5.5 months $[9,10]$. However, sorafenib is associated with a low response rate of $30 \%$ and a high risk of acquired drug resistance and disease progression, thus limiting its long-term clinical benefits [11]. Although other molecular targeted drugs have been developed, the targeted therapy efficacy in advanced HCC remains a serious concern.

Immune checkpoint inhibitors (ICls), which target cytotoxic T-lymphocyte antigen-4 (CTLA-4), programmed cell death protein1 (PD-1) and programmed cell death protein ligand 1 (PD-L1), show a promising prospect in cancer therapeutics [12]. The anti-PD-1 antibodies nivolumab and pembrolizumab have gained the U.S. Food and Drug Administration (FDA) approval as second-line agents for the treatment of advanced HCC [13, 14]. Despite the positive role of ICls for HCC, the subsequent phase 3 CheckMate459 [15] and KEYNOTE-240 [16] trials demonstrated that nivolumab (versus placebo) and pembrolizumab (versus sorafenib) failed to show significant survival superiority, indicating the necessity to explore more appropriate systemic treatment strategies to enhance the immunotherapy efficacy. The angiogenesis inhibitors can impair immunosuppression in the tumor microenvironment, thus facilitating antitumor efficacy of immune checkpoint inhibitors through influence on T cell activation, which provides a strong rationale for combination trials [17]. Recently, the combination of atezolizumab plus bevacizumab was approved by FDA as first-line therapy for unresectable HCC [18], which encourages further investigation for other potential promising combination treatment.

Hence, the present study aimed to evaluate the safety and therapeutic efficacy of Camrelizumab plus sorafenib in comparison with sorafenib monotherapy for patients with advanced HCC.

\section{Methods}

\subsection{Patients}

100 participants with advanced HCC who received sorafenib treatment from January 2019 to January 2021 in the Second Affiliated Hospital of Army Medical University were included in the study. The patient inclusion criteria were: (1) male or female patients aged $\geq 18$ years; (2) HCC diagnosis was based on histological examination or the criteria of the American Association for the Study of Liver Diseases (AASLD) guidelines [ $\left.{ }^{19}\right]$; (3) liver function of Child-Pugh class A or B; (4) Eastern Cooperative Oncology Group (ECOG) performance status score of 0 or 1; (5) presence of unresectable or metastatic lesions; (6) acceptable heart, hepatic, renal and hematologic functions ; (7) estimated life expectancy $\geq 12$ weeks; (8) at least one measurable lesion according to the modified Response Evaluation Criteria in Solid Tumors (mRECIST) [ $\left.{ }^{20}\right]$. The following patients were excluded: (1) a previous history of sorafenib or any other PD-L1/PD-1 antagonist treatment; (2) other malignant tumors; (3) pregnancy or breastfeeding; and (4) Patients with incomplete follow-up data. The preoperative biochemical and radiological examinations were routinely performed in all patients. The study was approved by the Ethics Committee of the Second Affiliated Hospital of Army Medical University. Written consent was obtained from patients for the collection of clinical data for research purposes.

\subsection{Treatment Protocol}


The sorafenib-only group was administered sorafenib $400 \mathrm{mg}$ orally twice daily. The combined-therapy group received camrelizumab $200 \mathrm{mg}$ intravenously every 2 weeks in combination with sorafenib $400 \mathrm{mg}$ orally once daily. When the patients experienced grade $3 / 4$ treatment-related adverse events (AEs), the dose of sorafenib was reduced to $200 \mathrm{mg}$ /day or discontinued until the AEs severity decreased to grade $\leq 2$. Patients were treated until death, disease progression, unacceptable toxicity, consent withdrawn from the study.

\subsection{Endpoints And Assessments}

Demographic and clinical data were recorded and included age, gender, hepatitis B virus (HBV) carrier, Liver cirrhosis, ECOG performance score, and ChildPugh score, Barcelona Clinic Liver Cancer (BCLC) stage, alpha-fetoprotein (AFP), total bilirubin (TBIL), albumin (ALB), aspartate aminotransferase (AST), alanine aminotransferase (ALT), platelet count (PLT), white blood cell (WBC), prothrombin time (PT), tumor size, tumor number, macrovascular invasion, extrahepatic metastasis, and previous local regional therapy. The patients received CT or MRI evaluation at baseline and every 2 cycles of treatment ( 8 weeks) thereafter. Tumor responses were assessed according to the mRECIST and classified as complete response (CR), partial response (PR), stable disease (SD), progressive disease (PD).

The primary endpoint was progression-free survival (PFS). Secondary endpoints included over survival (OS), objective response rate (ORR), disease control rate (DCR) and AEs.

PFS was calculated as the time from the start of treatment to the date of disease progression or death due to any cause. OS was defined as the time from the start of treatment to the date of death due to any cause or the last follow-up. DCR was defined as the proportion of patients with CR, PR, and SD. ORR was defined as the proportion of patients with CR or PR. AEs were assessed and graded based on the Common Terminology Criteria for Adverse Events (CTCAE, version 4.0).

\subsection{Statistical Analysis}

We performed Propensity score matching (PSM) analysis based on the following variables: age, sex, HBV carrier, liver cirrhosis, ECOG performance score, Child-Pugh stage, BCLC stage, AFP, tumor size, tumor number, macrovascular invasion, extrahepatic metastasis and previous local regional therapy. The continuous data were expressed as mean \pm standard deviation or median with interquartile range and compared using t test or Mann-Whitney-Wilcoxon test. The categorical data were presented as frequency with proportion and analysed using Chi-square test or Fisher exact test. OS and PFS were estimated by the Kaplan-Meier method and log-rank test. P values $<0.05$ were considered statistically significant. Statistical analyses and PSM were conducted using SPSS version 25.0 (IBM SPSS, Inc, Chicago, IL).

\section{Results}

\subsection{Baseline}

Between January 2019 and January 2021, a total of 100 patients with advanced HCC in our hospital were enrolled in the present study, of which 35 patients received combined therapy and 65 patients received sorafenib monotherapy. 34 pairs were matched after PSM. The patient characteristics at baseline are shown in Table 1. No significant differences were found between the two groups in age, sex, HBV carrier, liver cirrhosis, ECOG performance score, Child-Pugh stage, BCLC stage, AFP, TB, ALB, AST, ALT, PLT, WBC, PT, tumor size, tumor number, macrovascular invasion, extrahepatic metastasis and previous local regional therapy both before and after PSM. 
Table 1

The baseline patient characteristics

\begin{tabular}{|c|c|c|c|c|c|c|}
\hline Variables & Before PSM & & & After PSM & & \\
\hline combined-therapy group & $\begin{array}{l}\text { sorafenib-only } \\
\text { group }\end{array}$ & $P$ & $\begin{array}{l}\text { combined-therapy } \\
\text { group }\end{array}$ & $\begin{array}{l}\text { sorafenib-only } \\
\text { group }\end{array}$ & $P$ & \\
\hline$n=35$ & $n=65$ & & $\mathrm{n}=34$ & $\mathrm{n}=34$ & & \\
\hline Age,years & $53.0 \pm 10.3$ & $53.2 \pm 11.6$ & 0.917 & $53.1 \pm 10.4$ & $53.3 \pm 9.3$ & 0.922 \\
\hline Sex(Male: Female) & $32: 3$ & $54: 11$ & 0.398 & $31: 3$ & $31: 3$ & 1.000 \\
\hline HBV carrier & $33(94.3 \%)$ & $52(80.0 \%)$ & 0.106 & $32(94.1 \%)$ & $33(97.1 \%)$ & 1.000 \\
\hline Liver cirrhosis & $20(57.1 \%)$ & $41(63.1 \%)$ & 0.562 & $20(58.8 \%)$ & $25(73.5 \%)$ & 0.200 \\
\hline ECOG performance score & & & 0.723 & & & 0.742 \\
\hline 0 & $7(20.0 \%)$ & $15(23.1 \%)$ & & $6(17.6 \%)$ & $5(14.7 \%)$ & \\
\hline 1 & $28(80.0 \%)$ & $50(76.9 \%)$ & & $28(82.4 \%)$ & $29(85.3 \%)$ & \\
\hline Child-Pugh stage & & & 0.485 & & & 1.000 \\
\hline A & $2(5.7 \%)$ & $8(12.3 \%)$ & & $2(5.9 \%)$ & $3(8.8 \%)$ & \\
\hline B & $33(94.3 \%)$ & $57(87.7 \%)$ & & $32(94.1 \%)$ & $31(91.2 \%)$ & \\
\hline BCLC stage & & & 0.364 & & & 0.417 \\
\hline B & $11(31.4 \%)$ & $15(23.1 \%)$ & & $11(32.4 \%)$ & $8(23.5 \%)$ & \\
\hline C & $24(68.6 \%)$ & $50(76.9 \%)$ & & $23(67.6 \%)$ & $26(76.5 \%)$ & \\
\hline AFP & & & 0.950 & & & 0.462 \\
\hline$<400 \mathrm{ng} / \mathrm{mL}$ & $17(48.6 \%)$ & $32(49.2 \%)$ & & $16(47.1 \%)$ & $13(38.2 \%)$ & \\
\hline$\geq 400 \mathrm{ng} / \mathrm{mL}$ & $18(51.4 \%)$ & $33(50.8 \%)$ & & $18(52.9 \%)$ & $21(61.8 \%)$ & \\
\hline TBIL, $\mu \mathrm{mol} / \mathrm{L}$ & $20.0(12.2-29.7)$ & 19.2(13.5-31.1) & 0.883 & $20.0(12.2-29.7)$ & $18.1(14.1-30.7)$ & 0.695 \\
\hline$A L B, g / L$ & $38.9 \pm 4.6$ & $39.1 \pm 5.4$ & 0.809 & $38.7 \pm 4.5$ & $39.8 \pm 4.8$ & 0.348 \\
\hline AST, IU/L, & $56.6(44.8-98.2)$ & $74.1(47.9-105.6)$ & 0.152 & $56.6(44.8-98.2)$ & $74.4(48.2-112.2)$ & 0.336 \\
\hline ALT, IU/L & $52.5(40.7-65.2)$ & $48.8(36.4-66.9)$ & 0.968 & $52.5(40.7-65.2)$ & $43.6(34.9-66.0)$ & 0.300 \\
\hline $\operatorname{PLT}\left(10^{9} / \mathrm{L}\right)$ & $127.0(81.0-181.0)$ & $\begin{array}{l}145.0(86.0- \\
200.0)\end{array}$ & 0.432 & $127.0(81.0-181.0)$ & $\begin{array}{l}163.5(86.8- \\
221.0)\end{array}$ & 0.149 \\
\hline WBC $\left(10^{9} / \mathrm{L}\right)$ & $5.5(4.4-8.4)$ & $6.0(4.3-7.5)$ & 0.900 & $5.5(4.4-8.4)$ & $6.2(5.0-7.6)$ & 0.349 \\
\hline PT (second) & $12.0(11.4-12.8)$ & $12.0(11.3-12.7)$ & 0.696 & $12.0(11.4-12.8)$ & $12.0(11.3-12.6)$ & 0.606 \\
\hline Tumor size, cm & $6.7 \pm 3.6$ & $7.5 \pm 4.1$ & 0.318 & $6.8 \pm 3.6$ & $8.2 \pm 4.6$ & 0.200 \\
\hline Tumor number & & & 0.316 & & & 0.618 \\
\hline Solitary & $12(34.3 \%)$ & $29(44.6 \%)$ & & $12(35.3 \%)$ & $14(41.2 \%)$ & \\
\hline Multiple & $23(65.7 \%)$ & $36(55.4 \%)$ & & $22(64.7 \%)$ & $20(58.8 \%)$ & \\
\hline Macrovascular invasion & $15(42.9 \%)$ & $33(50.8 \%)$ & 0.450 & $15(44.1 \%)$ & $19(55.9 \%)$ & 0.332 \\
\hline Extrahepatic metastasis & $22(62.9 \%)$ & $40(61.5 \%)$ & 0.897 & $21(61.8 \%)$ & $23(67.6 \%)$ & 0.612 \\
\hline \multicolumn{7}{|l|}{$\begin{array}{l}\text { Previous local regional } \\
\text { therapy }\end{array}$} \\
\hline Surgery & $11(31.4 \%)$ & $11(16.9 \%)$ & 0.095 & $10(29.4 \%)$ & $8(23.5 \%)$ & 0.582 \\
\hline Ablation & $1(2.9 \%)$ & $2(3.1 \%)$ & 0.950 & $1(2.9 \%)$ & $1(2.9 \%)$ & 1.000 \\
\hline TACE & $18(47.4 \%)$ & $33(50.8 \%)$ & 1.000 & $17(50.0 \%)$ & $20(58.8 \%)$ & 0.465 \\
\hline
\end{tabular}


No CR was observed in either group (Table 2). Before PSM, the ORR was significantly higher in the combined-therapy group than in the sorafenib-only group (17.1\% vs. $3.1 \%, P=0.037$ ). The DCR was $68.6 \%$ in the combined-therapy group and $72.3 \%$ in the sorafenib-only group, respectively $(P=0.695)$. There was no significant difference in OS between the two groups, with median OS of 14.1 months (6.8-21.4 months) in the combined-therapy group and 9.6 months (6.712.5 months $)$ in the sorafenib-only group ( $P=0.135)$. However, the combined-therapy group exhibited statistically significant prolonged $P F S$ compared to the sorafenib-only group (10.2 months; $95 \% \mathrm{Cl} 4.5-19.0$ vs. 6.1 months; $95 \% \mathrm{Cl} 2.5-9.7 ; \mathrm{P}=0.041)$ (Fig. 1).

Table 2

Tumor responses for patients with advanced hepatocellular carcinoma

\begin{tabular}{|lllllll|}
\hline Response & Before PSM & & After PSM & \\
\cline { 2 - 6 } & combined-therapy group & sorafenib-only group & $\boldsymbol{P}$ & combined-therapy group & sorafenib-only group & $\boldsymbol{P}$ \\
\hline Objective response & $6(17.1 \%)$ & $2(3.1 \%)$ & 0.037 & $6(17.6 \%)$ & $0(0.0 \%)$ & 0.010 \\
\hline Disease control & $24(68.6 \%)$ & $47(72.3 \%)$ & 0.695 & $24(70.6 \%)$ & 1.000 \\
\hline Complete response & $0(0.0 \%)$ & $0(0.0 \%)$ & 1.000 & $0(0.0 \%)$ & $0(0.0 \%)$ & $0(0.0 \%)$ \\
\hline Partial response & $6(17.1 \%)$ & $2(3.1 \%)$ & 0.037 & $6(17.6 \%)$ & 0.010 \\
\hline Stable disease & $18(51.4 \%)$ & $45(69.2 \%)$ & 0.079 & $18(52.9 \%)$ & $24(70.6 \%)$ & $10(29.4 \%)$ \\
\hline Progressive disease & $11(31.4 \%)$ & $18(27.7 \%)$ & 0.695 & $10(29.4 \%)$ & 1.000 \\
\hline
\end{tabular}

The similar results were observed after PSM. The ORR was $17.6 \%$ among patients who received combined-therapy and $0.0 \%$ among patients who received sorafenib-only therapy $(P=0.010)$. The DCR were $70.6 \%$ in the combined-therapy group and the sorafenib-only group $(P=1.000)$. The median 0 S were comparable in the combined-therapy group and sorafenib-only group (14.1 months; $95 \% \mathrm{Cl} 7.2-21.0$ vs. 9.6 months; $95 \% \mathrm{Cl} 6.1-13.1 ; \mathrm{P}=0.105)$. The median PFS in the combined-therapy group $(9.5$ months; $95 \% \mathrm{Cl} 1.2-17.8)$ were longer than those in the sorafenib-only group $(4.7 \mathrm{months} ; 95 \% \mathrm{Cl} 1.6-7.8)(\mathrm{P}=$ 0.043).

\subsection{Adverse Events}

All recorded treatment-related AEs are listed in Table 3. The most common AEs were hand and foot syndrome, thrombocytopenia and hyperbilirubinaemia in the combined-therapy group and hand and foot syndrome in the sorafenib-only group. Elevated transaminase was the most frequent grade $3 / 4$ AEs observed in both groups (before and after PSM). The incidence of thrombocytopenia $(p=0.005)$ and anemia $(p=0.040)$ before PSM and thrombocytopenia $(p=0.011)$ after PSM was significantly higher in the combined-therapy group than the sorafenib-only group. However, most of these AEs were grade 1 or 2 , which can be easily alleviated after dose adjustment and supportive treatment. Dose modifications or treatment interruptions due to AEs were similar in the combinedtherapy group and the sorafenib-only group ( $42.9 \%$ vs. $41.5 \%, \mathrm{P}=0.899$ before $\mathrm{PSM} ; 42.9 \%$ vs. $41.2 \%, \mathrm{P}=0.806$ after $\mathrm{PSM}$ ) . No treatment-associated deaths occurred in this study. 
Table 3

Treatment related adverse events

\begin{tabular}{|c|c|c|c|c|c|c|c|c|c|c|c|}
\hline \multirow[t]{3}{*}{ Adverse events } & \multicolumn{6}{|c|}{ Before PSM } & \multicolumn{5}{|l|}{ After PSM } \\
\hline & \multicolumn{2}{|c|}{$\begin{array}{l}\text { combined-therapy } \\
\text { group }\end{array}$} & \multicolumn{2}{|c|}{ sorafenib-only group } & \multicolumn{2}{|l|}{$P$} & \multicolumn{2}{|c|}{$\begin{array}{l}\text { combined-therapy } \\
\text { group }\end{array}$} & \multicolumn{2}{|c|}{ sorafenib-only group } & \multirow{2}{*}{$\begin{array}{l}P \\
\begin{array}{l}\text { Any } \\
\text { gradı }\end{array}\end{array}$} \\
\hline & Any grade & $\begin{array}{l}\text { Grade 3- } \\
4\end{array}$ & Any grade & $\begin{array}{l}\text { Grade 3- } \\
4\end{array}$ & $\begin{array}{l}\text { Any } \\
\text { grade }\end{array}$ & $\begin{array}{l}\text { Grade } \\
3-4\end{array}$ & Any grade & $\begin{array}{l}\text { Grade 3- } \\
4\end{array}$ & Any grade & $\begin{array}{l}\text { Grade 3- } \\
4\end{array}$ & \\
\hline All & $33(94.3 \%)$ & $14(40.0 \%)$ & $59(90.8 \%)$ & $27(41.5 \%)$ & 0.817 & 0.881 & $32(94.1 \%)$ & $14(41.2 \%)$ & $30(88.2 \%)$ & $13(38.2 \%)$ & $0.66 c$ \\
\hline $\begin{array}{l}\text { Hand and foot } \\
\text { syndrome }\end{array}$ & $21(60.0 \%)$ & $2(5.7 \%)$ & $34(52.3 \%)$ & $4(6.2 \%)$ & 0.461 & 1.000 & $20(58.8 \%)$ & $2(5.9 \%)$ & $16(47.1 \%)$ & $0(0.0 \%)$ & 0.331 \\
\hline Hypertension & $3(8.6 \%)$ & $0(0.0 \%)$ & $8(12.3 \%)$ & $2(3.1 \%)$ & 0.815 & 0.765 & $3(8.8 \%)$ & $0(0.0 \%)$ & $3(8.8 \%)$ & $1(2.9 \%)$ & $1.00 \mathrm{C}$ \\
\hline Diarrhea & $10(28.6 \%)$ & $4(11.4 \%)$ & $23(35.4 \%)$ & $2(3.1 \%)$ & 0.489 & 0.216 & $9(26.5 \%)$ & $4(11.8 \%)$ & $13(38.2 \%)$ & $1(2.9 \%)$ & $0.30 C$ \\
\hline Rash & $8(22.9 \%)$ & $1(2.9 \%)$ & $7(10.8 \%)$ & $1(1.5 \%)$ & 0.106 & 1.000 & $7(20.6 \%)$ & $1(2.9 \%)$ & $3(8.8 \%)$ & $1(2.9 \%)$ & 0.171 \\
\hline Fatigue & $8(22.9 \%)$ & $0(0.0 \%)$ & $18(27.7 \%)$ & $0(0.0 \%)$ & 0.599 & 1.000 & $7(20.6 \%)$ & $0(0.0 \%)$ & $7(20.6 \%)$ & $0(0.0 \%)$ & $1.00 \mathrm{C}$ \\
\hline Abdominal pain & $4(11.4 \%)$ & $0(0.0 \%)$ & $12(18.5 \%)$ & $3(4.6 \%)$ & 0.360 & 0.499 & $4(11.8 \%)$ & $0(0.0 \%)$ & $5(14.7 \%)$ & $0(0.0 \%)$ & $1.00 \mathrm{C}$ \\
\hline Nausea/Vomiting & $3(8.6 \%)$ & $0(0.0 \%)$ & $5(7.7 \%)$ & $0(0.0 \%)$ & 1.000 & 1.000 & $3(8.8 \%)$ & $0(0.0 \%)$ & $1(2.9 \%)$ & $0(0.0 \%)$ & $0.60 \epsilon$ \\
\hline Fever & $2(5.7 \%)$ & $1(2.9 \%)$ & $4(6.2 \%)$ & $0(0.0 \%)$ & 1.000 & 0.752 & $2(5.9 \%)$ & $1(2.9 \%)$ & $1(2.9 \%)$ & $0(0.0 \%)$ & 1.000 \\
\hline Cough & $2(5.7 \%)$ & $0(0.0 \%)$ & $3(4.6 \%)$ & $0(0.0 \%)$ & 1.000 & 1.000 & $2(5.9 \%)$ & $0(0.0 \%)$ & $1(2.9 \%)$ & $0(0.0 \%)$ & $1.00 \mathrm{C}$ \\
\hline $\begin{array}{l}\text { Gingival } \\
\text { hemorrhage }\end{array}$ & $3(8.6 \%)$ & $0(0.0 \%)$ & $2(3.1 \%)$ & $0(0.0 \%)$ & 0.471 & 1.000 & $3(8.8 \%)$ & $0(0.0 \%)$ & $1(2.9 \%)$ & $0(0.0 \%)$ & $0.60 \epsilon$ \\
\hline $\begin{array}{l}\text { Elevated } \\
\text { transaminase }\end{array}$ & $11(31.4 \%)$ & $5(14.3 \%)$ & $13(20.0 \%)$ & $8(12.3 \%)$ & 0.202 & 1.000 & $11(32.4 \%)$ & $5(14.7 \%)$ & $7(20.6 \%)$ & $5(14.7 \%)$ & $0.41 C$ \\
\hline Hyperbilirubinaemia & $14(40.0 \%)$ & $4(11.4 \%)$ & $19(29.2 \%)$ & $7(10.8 \%)$ & 0.275 & 1.000 & $14(41.2 \%)$ & $4(11.8 \%)$ & $13(38.2 \%)$ & $5(14.7 \%)$ & $0.80<$ \\
\hline Leukopenia & $8(22.9 \%)$ & $1(2.9 \%)$ & $8(12.3 \%)$ & $1(1.5 \%)$ & 0.170 & 1.000 & $8(23.5 \%)$ & $1(2.9 \%)$ & $3(8.8 \%)$ & $0(0.0 \%)$ & $0.10 \mathrm{C}$ \\
\hline Thrombocytopenia & $17(48.6 \%)$ & $1(2.9 \%)$ & $14(21.5 \%)$ & $2(3.1 \%)$ & 0.005 & 1.000 & $17(50.0 \%)$ & $1(2.9 \%)$ & $7(20.6 \%)$ & $1(2.9 \%)$ & 0.011 \\
\hline Anemia & $13(37.1 \%)$ & $0(0.0 \%)$ & $12(18.5 \%)$ & $3(4.6 \%)$ & 0.040 & 0.105 & $13(38.2 \%)$ & $0(0.0 \%)$ & $6(17.6 \%)$ & $3(8.8 \%)$ & 0.05 ک \\
\hline
\end{tabular}

\section{Discussion}

The continuous emergence of new agents for systemic treatment options represented a major breakthrough in the management of advanced HCC. Although the multikinase inhibitor sorafenib has been was approved as the first-line systemic treatment against advanced HCC for a decade, its survival benefit is limited and response rate is low [9]. Recently, the combined molecular targeted therapy with immunotherapy attracted tremendous interests due to the potential improved therapeutic efficacy compared with monotherapy [21,22]. Our study is the first, to our knowledge, to analyze the efficacy and safety of camrelizumab and sorafenib in patients with advanced HCC. The result demonstrated that the combined therapy showed superiority over sorafenib monotherapy in terms of PFS and ORR, though the OS benefit was not observed.

Different combined treatment modalities have been discussed in the advanced HCC due to the limited clinical benefits of monotherapy [23]. The recent introduction of immunotherapy demonstrated promising efficacy in solid tumor treatment and various clinical trials involving immunotherapy are currently ongoing to explore the potential survival benefit in HCC patients [24,25]. However, the optimal combined regimens remain undefined in spite of the remarkable progress in systemic therapy of advanced HCC. So far, there is little knowledge of the potential synergic effects on the combination of camrelizumab and sorafenib in patients with advanced HCC.

In our present study, treatment efficacy of camrelizumab plus sorafenib was assessed in the 34 patients with advanced HCC, observing an ORR of $17.6 \%$, a DCR of $70.6 \%$, a median PFS of 9.5 months and a median OS of 14.1 months. There are limited clinical trials involving combination therapies of immune checkpoint inhibitors and molecular targeted agents in HCC. In a phase II trial,Camrelizumab combined with apatinib showed median PFS of 5.7 months and 5.5 months and ORR of $34.3 \%$ and $22.5 \%$ in the first-line and the second-line treatment for advanced HCC, respectively [26]. In a phase lb study, lenvatinib plus pembrolizumab showed a median PFS of 8.6 months, a median OS of 22 months and an ORR of $46 \%$ in patients with unresectable HCC [27]. In the phase III IMbrave150 study [18], patients with unresectable HCC in the atezolizumab-bevacizumab group showed better clinical outcomes compared to sorafenib, with a median PFS of 6.8 months and an ORR of $89 \%$. The possible reasons for discrepancies were related to the retrospective observational design, differences in patient baseline characteristics and different treatment regimens. In our study, $44.1 \%$ had macrovascular invasion and $61.8 \%$ had extrahepatic metastasis $52.9 \%$ had a baseline AFP $>400 \mathrm{ng} / \mathrm{ml}$, while only $29.4 \%$ had received surgery. Lacking of effective post-progression therapy could be another explanation for the relatively short OS in our study. Although limitations should be considered regarding the interpretation of the results, our findings provided insight into potential therapeutic strategies for advanced HCC. 
Despite the great promise of molecular targeted agents, their clinical benefits are limited regarding tumoral heterogeneity and acquired resistance [28, 29], emphasizing the necessity of exploring combination therapies to improve the therapeutic efficacy. Our findings showed the addition of camrelizumab to sorafenib was associated with prolonged PFS and higher ORR, which indicated that the combination of immunotherapy and targeted therapy was associated with enhanced antitumor benefit. Preclinical studies demonstrated that antiangiogenic agents targeting VEGF/VEGFR could inhibit tumor growth and metastasis $[29,30]$. In addition, angiogenesis inhibitors possessed immunomodulatory effects including increasing T-cell activity and promoting T-cell infiltration [31]. On the other hand, vasculature normalization via inhibition of angiogenesis could reduce tumor hypoxia and improve drug delivery, and facilitates immune cell infiltration [32]. Therefore, the targeted therapy could reprogram the immunosuppressive tumor microenvironment into an immunostimulatory environment, thereby contributing to enhancing antitumor immunity. 17 Moreover, more studies are encouraged to investigate the underlying mechanism of enhanced antitumor effects and identify patients who will benefit most from the combination.

The most common sorafenib-related AEs were in accordance with those observed in the previous reports. Here, any-grade AEs of thrombocytopenia occurred more frequently in the combined-therapy group compared with the sorafenib-only group, which were related to utilization of camrelizumab. Given the increased hematologic toxicities in the combination therapy, the incidence of grade $3 / 4$ toxicities was comparable between the two treatment groups. Moreover, most of the AEs in both groups were mild to moderate in severity and no significant difference in the incidence of dose adjustments or treatment interruptions was observed between the two groups. The current study showed that the side effects of combined therapy are generally controllable and tolerable.

Several limitations in this study need to be addressed. First, the retrospective design of the retrospective study may have introduced potential biases, though PSM was introduced to reduce potential selection bias. Second, the study was based on a single-center experience with a relatively small number of patients. Third, the follow-up period was short. Furthermore, the heterogeneous individual therapeutic response highlights the need to understand who will respond better to the treatment.

\section{Conclusion}

In conclusion, camrelizumab combined with sorafenib appears to be a promising therapeutic strategy in the management of advanced HCC, with prolonged PFS, higher ORR and well-tolerated AEs. The results offer our preliminary experience in combination strategies for advanced HCC, which are informative for clinical decision making. Nevertheless, further prospective randomized controlled studies with larger sample size and longer follow-up time are warranted to support these preliminary findings of the study.

\section{Declarations}

\section{Ethnics declarations}

\section{Ethics approval and consent to participate}

The study was approved by the Ethics Committee of the Second Affiliated Hospital of Army Medical University. Written consent was obtained from patients for the collection of clinical data for research purposes.

\section{Consent for publication}

Not applicable.

\section{Availability of data and materials}

The data and material are available for reasonable requests.

\section{Competing interests}

The authors declare that they have no conflicts of interest.

\section{Funding}

This study was supported by the Medical Research Project jointly funded by Chongqing Science and Technology Commission and Chongqing Health Commission (2019ZDXM046), the Technological Innovation and Application Demonstration Special Project of Chongqing (cstc2018jscx-mszdX0012), and the Scientific and Technological Innovation Special Project of Army Medical University (2019XLC2006).

\section{Authors' Contributions}

Qinqin Liu, Jing Li, Nan You, Ke Wu, and Lu Zheng contributed to the study conception and design. Qinqin Liu, Jing Li, Nan You, Ke Wu and Xuehui Peng collected the data. Qinqin Liu, Jing Li and Nan You analyzed the data. Qinqin Liu drafted the manuscript, and the other authors revised the manuscript. All authors reviewed and approved the manuscript.

\section{Acknowledgements}

Not applicable. 


\section{References}

1. Jemal A, Bray F, Center MM, Ferlay J, Ward E, Forman D (2011) Global cancer statistics. CA Cancer J Clin 61:69-90. https://doi.org/10.3322/caac.20107

2. Bruix J, Gores GJ, Mazzaferro V (2014) Hepatocellular carcinoma: clinical frontiers and perspectives. Gut 63(5):844-855. https://doi.org/10.1136/gutjnl2013-306627

3. Association for the Study of the Liver (2018) EASL Clinical Practice Guidelines: Management of hepatocellular carcinoma. J Hepatol 69:182236.https://doi.org/10.1016/j.jhep.2018.03.019

4. She WH, Chok K (2015) Strategies to increase the resectability of hepatocellular carcinoma. World J hepatol 7:2147-2154. https://doi.org/10.4254/wjh.v7.i18.2147

5. Abou-Alfa GK, Meyer T, Cheng AL et al (2018) Cabozantinib in Patients with Advanced and Progressing Hepatocellular Carcinoma. N Engl J Med 379:5463.https://doi.org/10.1056/NEJMoa1717002

6. Zhao Y, Zhang YN, Wang KT, Chen L (2020) Lenvatinib for hepatocellular carcinoma: From preclinical mechanisms to anti-cancer therapy. Biochim Biophys Acta Rev Cancer 1874:188391. https://doi.org/10.1016/j.bbcan.2020.188391

7. Finn RS, Merle P, Granito A et al (2018) Outcomes of sequential treatment with sorafenib followed by regorafenib for HCC: Additional analyses from the phase III RESORCE trial. J Hepatol 69:353-358.https://doi.org/10.1016/j.jhep.2018.04.010

8. Liu Z, Lin Y, Zhang J et al (2019) Molecular targeted and immune checkpoint therapy for advanced hepatocellular carcinoma. J Exp Clin Cancer Res 38:447. https://doi.org/10.1186/s13046-019-1412-8

9. Llovet JM, Ricci S, Mazzaferro V et al (2008) Sorafenib in advanced hepatocellular carcinoma. N Engl J Med 359:378390.https://doi.org/10.1056/NEJMoa0708857

10. Cheng AL, Kang YK, Chen Z et al (2009) Efficacy and safety of sorafenib in patients in the Asia-Pacific region with advanced hepatocellular carcinoma: a phase III randomised, double-blind, placebo-controlled trial. Lancet Oncol 10:25-34. https://doi.org/10.1016/S1470-2045(08)70285-7

11. Heimbach JK, Kulik LM, Finn RS et al (2018) AASLD guidelines for the treatment of hepatocellular carcinoma. Hepatology 67:358-380. https://doi.org/10.1002/hep.29086

12. Federico P, Petrillo A, Giordano P et al (2020) Immune Checkpoint Inhibitors in Hepatocellular Carcinoma: Current Status and Novel Perspectives. Cancers 12. https://doi.org/10.3390/cancers12103025

13. El-Khoueiry AB, Sangro B, Yau T et al (2017) Nivolumab in patients with advanced hepatocellular carcinoma (CheckMate 040): an open-label, noncomparative, phase 1/2 dose escalation and expansion trial. Lancet 389:2492-2502. https://doi.org/10.1016/S0140-6736(17)31046-2

14. Zhu AX, Finn RS, Edeline $\mathrm{J}$ et al (2018) Pembrolizumab in patients with advanced hepatocellular carcinoma previously treated with sorafenib (KEYNOTE224): a non-randomised, open-label phase 2 trial. Lancet Oncol 19:940-952. https://doi.org/10.1016/S1470-2045(18)30351-6

15. Yau T, Park J, Finn R, et al. CheckMate 459: A randomized, multi-center phase III study of nivolumab (NIVO) vs sorafenib (SOR) as first-line (1L) treatment in patients (pts) with advanced hepatocellular carcinoma (aHCC). Annals of Oncology. 2019;30:v874-v875. https://doi.org/10.1093/annonc/mdz394.029

16. Finn RS, Ryoo B-Y, Merle P et al (2019) Results of KEYNOTE-240: phase 3 study of pembrolizumab (Pembro) vs best supportive care (BSC) for second line therapy in advanced hepatocellular carcinoma (HCC). American Society of Clinical Oncology https://doi.org/10.1200/JC0.2019.37.15_suppl.4004

17. Kudo M (2020) Scientific Rationale for Combined Immunotherapy with PD-1/PD-L1 Antibodies and VEGF Inhibitors in Advanced Hepatocellular Carcinoma. Cancers 12(5). https://doi.org/10.3390/cancers12051089

18. Finn RS, Qin S, Ikeda M et al (2020) Atezolizumab plus Bevacizumab in Unresectable Hepatocellular Carcinoma. N Engl J Med 382:1894-1905. https://doi.org/10.1056/NEJMoa1915745

19. Marrero JA, Kulik LM, Sirlin CB et al (2018) Diagnosis, Staging, and Management of Hepatocellular Carcinoma: 2018 Practice Guidance by the American Association for the Study of Liver Diseases. 68:723-750. https://doi.org/10.1002/hep.29913

20. Lencioni R, Llovet JM (2010) Modified RECIST (mRECIST) assessment for hepatocellular carcinoma. Semin Liver Dis 30:52-60. https://doi.org/10.1055/s0030-1247132

21. El Dika I, Khalil DN, Abou-Alfa GK (2019) Immune checkpoint inhibitors for hepatocellular carcinoma. Cancer 125:3312-3319. https://doi.org/10.1002/cncr.32076

22. Hilmi M, Neuzillet C, Calderaro J, Lafdil F, Pawlotsky JM, Rousseau B (2019) Angiogenesis and immune checkpoint inhibitors as therapies for hepatocellular carcinoma: current knowledge and future research directions. J Immunother Cancer 7:333. https://doi.org/10.1186/s40425-019-0824-5

23. Zheng L, Fang S, Wu F et al (2020) Efficacy and Safety of TACE Combined With Sorafenib Plus Immune Checkpoint Inhibitors for the Treatment of Intermediate and Advanced TACE-Refractory Hepatocellular Carcinoma: A Retrospective Study. Front Mol Biosci 7:609322.

https://doi.org/10.3389/fmolb.2020.609322

24. Tsang J, Wong JSL, Kwok GGW et al (2021) Nivolumab + Ipilimumab for patients with hepatocellular carcinoma previously treated with Sorafenib. Expert Rev Gastroenterol \& Hepatol 1-10. https://doi.org/10.1080/17474124.2021.1899808

25. Liu X, Qin S (2019) Immune Checkpoint Inhibitors in Hepatocellular Carcinoma: Opportunities and Challenges. Oncologist 24:S3-s10. https://doi.org/10.1634/theoncologist.2019-IO-S1-s01

26. Xu J, Shen J, Gu S et al (2021) Camrelizumab in Combination with Apatinib in Patients with Advanced Hepatocellular Carcinoma (RESCUE): A Nonrandomized, Open-label, Phase II Trial 27:1003-1011. https://doi.org/10.1158/1078-0432.CCR-20-2571

27. Finn RS, Ikeda M, Zhu AX et al (2020) Phase lb Study of Lenvatinib Plus Pembrolizumab in Patients With Unresectable Hepatocellular Carcinoma. J Clin oncol 38:2960-2970. https://doi.org/10.1200/JC0.20.00808 
28. Chen S, Cao Q, Wen W, Wang H (2019) Targeted therapy for hepatocellular carcinoma: Challenges and opportunities. Cancer lett 460:1-9. https://doi.org/10.1016/ j.canlet. 2019.114428

29. Kudo M, Finn RS, Qin S et al (2018) Lenvatinib versus sorafenib in first-line treatment of patients with unresectable hepatocellular carcinoma: a randomised phase 3 non-inferiority trial. Lancet 391:1163-1173.https://doi.org/10.1016/S0140-6736(18)30207-1

30. Qin S, Li A, Yi M, Yu S, Zhang M, Wu K (2019) Recent advances on anti-angiogenesis receptor tyrosine kinase inhibitors in cancer therapy. J Hematol Oncol 12(1):27. https://doi.org/10.1186/s13045-019-0718-5

31. Yang J, Yan J, Liu B (2018) Targeting VEGF/VEGFR to Modulate Antitumor Immunity. Front Immunol 9:978. https://doi.org/10.3389/fimmu.2018.00978

32. Yi M, Jiao D, Qin S, Chu Q, Wu K, Li A (2019) Synergistic effect of immune checkpoint blockade and anti-angiogenesis in cancer treatment. Mol Cancer 18:60. https://doi.org/10.1186/s12943-019-0974-6

\section{Figures}
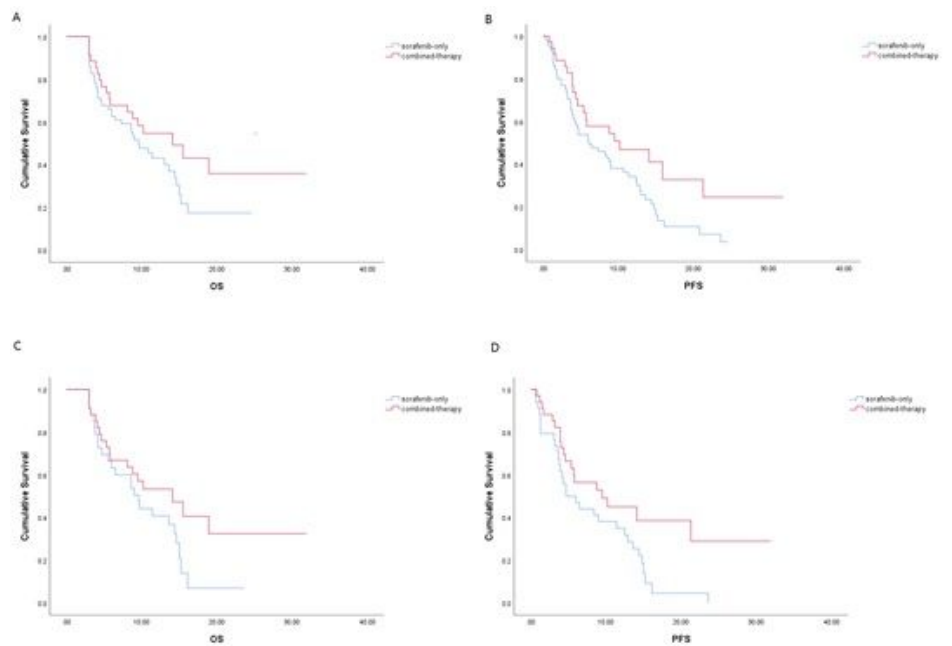

Figure 1

Kaplan-Meier survival curve. OS (A) and PFS (B) in the combined-therapy group and the sorafenib-only group before PSM. OS (C) and PFS (D) in the combined-therapy group and the sorafenib-only group before PSM. OS, overall survival; PFS, progression-free survival. 